\title{
Continuous extraction of black liquor salts under
}

\section{hydrothermal conditions}

\author{
Runyu Wang ${ }^{1,2}$, Roger Deplazes ${ }^{1,3}$, Frédéric Vogel ${ }^{1,4}$, and David Baudouin ${ }^{1 *}$. \\ ${ }^{1}$ Laboratory for Bioenergy and Catalysis, Paul Scherrer Institute (PSI), 5232 Villigen PSI, Switzerland \\ 2 State Key Laboratory of Multiphase Flow in Power Engineering (SKLMF), Xi'an Jiaotong University, \\ 28 Xianning West Road, Xi'an 710049, Shaanxi, China \\ ${ }^{3}$ School of Life Sciences, University of Applied Sciences Northwestern Switzerland (FHNW), 4132 \\ Muttenz, Switzerland \\ ${ }^{4}$ Institute of Biomass and Resource Efficiency, University of Applied Sciences Northwestern \\ Switzerland (FHNW), 5210 Windisch, Switzerland \\ *E-Mail: david.baudouin@psi.ch
}




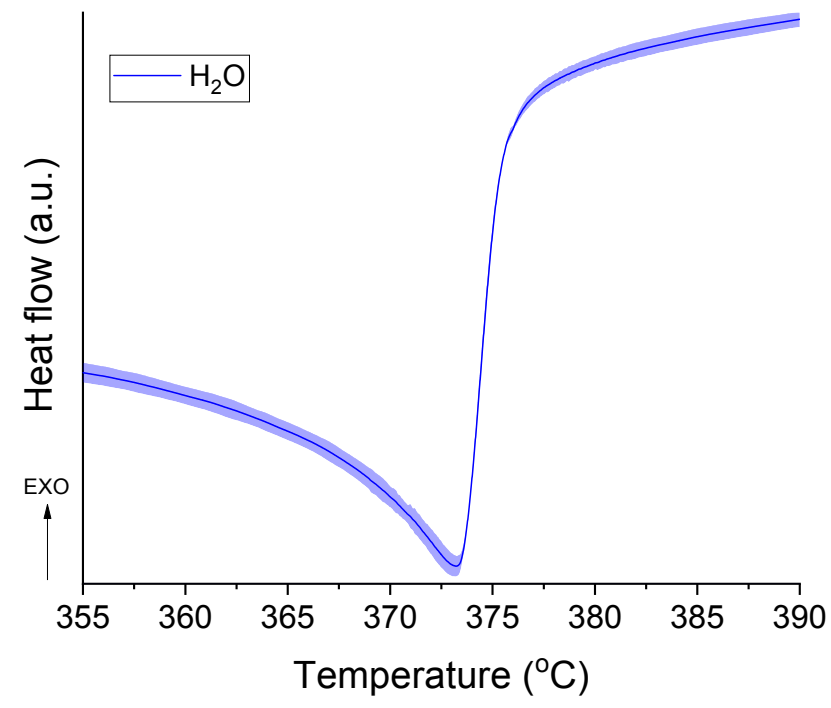

Figure S1. Reproducibility of HP-DSC study of $300 \mathrm{~kg} \mathrm{~m}^{-3}$ pure water sample at $1 \mathrm{~K} \mathrm{~min}^{-1}$. Blue line is the average number of three separate experiments, the shadow represents the standard deviation. (note the broad endothermic peak between 365 and $373^{\circ} \mathrm{C}$, which is not very pronounced at a heating ramp of $0.1 \mathrm{~K} \mathrm{~min}^{-1}$ ) 
Table S1. Mass balances and $\mathrm{pH}$ values for various continuous salt extraction experiments.

\begin{tabular}{|c|c|c|c|c|c|c|c|c|c|c|c|c|c|c|}
\hline \multirow[t]{2}{*}{ Element } & \multirow[t]{2}{*}{ Balance } & \multicolumn{13}{|c|}{$\mathrm{K} /(\mathrm{K}+\mathrm{Na})$ Ratio } \\
\hline & & $\begin{array}{c}0 \\
(39 \mathrm{~min})\end{array}$ & $\begin{array}{c}0.1 \\
(86 \mathrm{~min})\end{array}$ & $\begin{array}{c}0.2 \\
(77 \mathrm{~min})\end{array}$ & $\begin{array}{c}0.2 \\
(154 \mathrm{~min}) \\
\end{array}$ & $\begin{array}{c}0.2 \\
(213 \min ) \\
\end{array}$ & $\begin{array}{c}0.25 \\
101 \text { (min) }\end{array}$ & $\begin{array}{c}0.4 \\
(75 \mathrm{~min})\end{array}$ & $\begin{array}{c}0.4 \\
(138 \mathrm{~min})\end{array}$ & $\begin{array}{c}0.4 \\
(300 \mathrm{~min}) \\
\end{array}$ & $\begin{array}{c}0.5 \\
(88 \mathrm{~min})\end{array}$ & $\begin{array}{c}0.75 \\
(90 \mathrm{~min})\end{array}$ & $\begin{array}{c}0.75 \\
(170 \mathrm{~min}) \\
\end{array}$ & $\begin{array}{c}0.75 \\
(225 \mathrm{~min})\end{array}$ \\
\hline \multirow{4}{*}{$\mathrm{Na}(\mathrm{mg} / \mathrm{min})$} & Feed & $144 \pm 1$ & $98 \pm 3$ & $86 \pm 2$ & $83 \pm 2$ & $85 \pm 3$ & $79 \pm 1$ & & & & $52 \pm 1$ & $24 \pm 1$ & $29 \pm 1$ & $31 \pm 2$ \\
\hline & Desalinated & $1.5 \pm 0.2$ & $0.6 \pm 0.1$ & $0.4 \pm 0.1$ & $0.4 \pm 0.1$ & $0.4 \pm 0.1$ & $0.3 \pm 0.1$ & & & & $0.2 \pm 0.1$ & $0.1 \pm 0.1$ & $0.0 \pm 0.1$ & $0.1 \pm 0.1$ \\
\hline & Brine & $33 \pm 1$ & $73 \pm 3$ & $79 \pm 4$ & $61 \pm 3$ & $35 \pm 2$ & $76 \pm 4$ & & & & $53 \pm 3$ & $23 \pm 1$ & $28 \pm 1$ & $30 \pm 1$ \\
\hline & Recovery (\%) & $24 \pm 2$ & $75 \pm 4$ & $93 \pm 5$ & $73 \pm 4$ & $41 \pm 2$ & $96 \pm 5$ & & & & $99 \pm 5$ & $96 \pm 5$ & $97 \pm 4$ & $98 \pm 5$ \\
\hline \multirow[t]{4}{*}{$\mathrm{K}(\mathrm{mg} / \mathrm{min})$} & Feed & - & $18.6 \pm 0.7$ & $36 \pm 1$ & $35 \pm 1$ & $36 \pm 1$ & $45 \pm 2$ & & & & $89 \pm 3$ & $106 \pm 5$ & $127 \pm 4$ & $134 \pm 6$ \\
\hline & Desalinated & - & $0.4 \pm 0.2$ & $0.5 \pm 0.1$ & $0.5 \pm 0.1$ & $0.5 \pm 0.1$ & $0.5 \pm 0.1$ & & & & $0.7 \pm 0.1$ & $0.8 \pm 0.1$ & $1.0 \pm 0.1$ & $1.2 \pm 0.1$ \\
\hline & Brine & - & $16.9 \pm 0.8$ & $37 \pm 2$ & $26 \pm 2$ & $14 \pm 1$ & $44 \pm 2$ & & & & $84 \pm 4$ & $100 \pm 5$ & $121 \pm 5$ & $128 \pm 6$ \\
\hline & Recovery (\%) & - & $93 \pm 5$ & $104 \pm 5$ & $81 \pm 4$ & $42 \pm 3$ & $100 \pm 5$ & & & & $96 \pm 4$ & $95 \pm 5$ & $96 \pm 4$ & $97 \pm 5$ \\
\hline \multirow[t]{4}{*}{$\overline{\mathrm{S}(\mathrm{mg} / \mathrm{min})}$} & Feed & $45.1 \pm 0.7$ & $28.4 \pm 0.5$ & $28.6 \pm 0.5$ & $27.7 \pm 0.4$ & $28.3 \pm 0.4$ & $27.5 \pm 0.5$ & $26.7 \pm 0.4$ & $27.0 \pm 0.4$ & $27.0 \pm 0.4$ & $27.0 \pm 0.3$ & $23.5 \pm 0.5$ & $28.3 \pm 0.6$ & $29.8 \pm 0.4$ \\
\hline & Desalinated & $2.4 \pm 0.1$ & $2.7 \pm 0.1$ & $1.3 \pm 0.1$ & $2.6 \pm 0.1$ & $2.5 \pm 0.1$ & $2.3 \pm 0.1$ & $0.6 \pm 0.1$ & $1.0 \pm 0.1$ & $1.0 \pm 0.1$ & $1.0 \pm 0.1$ & $0.8 \pm 0.1$ & $1.8 \pm 0.1$ & $2.3 \pm 0.1$ \\
\hline & Brine & $8.9 \pm 0.2$ & $21 \pm 2$ & $26 \pm 2$ & $19.6 \pm 0.9$ & $10.4 \pm 0.8$ & $24 \pm 2$ & $24.6 \pm 0.9$ & $24.7 \pm 0.5$ & $25.5 \pm 0.8$ & $24.1 \pm 0.7$ & $21.0 \pm 0.7$ & $25.6 \pm 0.9$ & $26.5 \pm 0.6$ \\
\hline & Recovery (\%) & $25 \pm 2$ & $91 \pm 5$ & $95 \pm 3$ & $80 \pm 2$ & $46 \pm 2$ & $96 \pm 5$ & $94 \pm 4$ & $95 \pm 3$ & $98 \pm 5$ & $93 \pm 3$ & $93 \pm 3$ & $99 \pm 3$ & $100 \pm 3$ \\
\hline \multirow{4}{*}{$\begin{array}{c}\text { S2- } \\
(\mathrm{mg} / \text { min })\end{array}$} & Feed & $30 \pm 2$ & $19.3 \pm 0.8$ & $18.2 \pm 0.7$ & $17.6 \pm 0.7$ & $18.0 \pm 0.9$ & $19 \pm 2$ & $16.2 \pm 0.7$ & $15 \pm 2$ & $15.6 \pm 0.6$ & $17 \pm 1$ & $15 \pm 1$ & $17.1 \pm 0.8$ & $18.0 \pm 0.7$ \\
\hline & Desalinated & $2.0 \pm 0.3$ & $0.9 \pm 0.1$ & $0.6 \pm 0.1$ & $0.7 \pm 0.2$ & $0.8 \pm 0 . .2$ & $0.8 \pm 0.1$ & $0.7 \pm 0.1$ & $0.6 \pm 0.1$ & $0.9 \pm 0.1$ & $0.5 \pm 0.1$ & $0.5 \pm 0.2$ & $0.7 \pm 0.1$ & $0.9 \pm 0.1$ \\
\hline & Brine & $6.0 \pm 0.2$ & $18.1 \pm 0.7$ & $18.4 \pm 0.6$ & $15.1 \pm 0.5$ & $6.3 \pm 0.5$ & $18 \pm 1$ & $14.1 \pm 0.3$ & $14.8 \pm 0.5$ & $14.4 \pm 0.4$ & $16 \pm 1$ & $14.2 \pm 0.6$ & $16.9 \pm 0.7$ & $17.2 \pm 0.7$ \\
\hline & Recovery (\%) & $26 \pm 4$ & $99 \pm 5$ & $105 \pm 6$ & $90 \pm 5$ & $39 \pm 4$ & $100 \pm 9$ & $91 \pm 6$ & $98 \pm 7$ & $98 \pm 7$ & $100 \pm 10$ & $99 \pm 7$ & $102 \pm 7$ & $100 \pm 6$ \\
\hline \multirow[t]{4}{*}{$\mathrm{C}(\mathrm{mg} / \mathrm{min})$} & Feed & $17.3 \pm 0.1$ & $11.4 \pm 0.2$ & $12.3 \pm 0.2$ & $11.9 \pm 0.2$ & $12.2 \pm 0.2$ & $11.0 \pm 0.1$ & $12.4 \pm 0.4$ & $12.6 \pm 0.6$ & $12.6 \pm 0.5$ & $10.7 \pm 0.1$ & $8.4 \pm 0.1$ & $10.1 \pm 0.2$ & $10.7 \pm 0.1$ \\
\hline & Desalinated & $0.7 \pm 0.4$ & $0.1 \pm 0.1$ & $0.1 \pm 0.1$ & 0 & 0 & 0 & $0.1 \pm 0.1$ & $0.1 \pm 0.1$ & $0.1 \pm 0.1$ & $0.1 \pm 0.1$ & $0.1 \pm 0.1$ & $0.1 \pm 0.1$ & $0.1 \pm 0.1$ \\
\hline & Brine & $3.7 \pm 0.1$ & $9.4 \pm 0.2$ & $9.5 \pm 0.1$ & $7.5 \pm 0.1$ & $4.4 \pm 0.1$ & $9.6 \pm 0.2$ & $11.0 \pm 0.3$ & $11.1 \pm 0.7$ & $11.0 \pm 0.4$ & $10.7 \pm 0.2$ & $7.6 \pm 0.1$ & $9.2 \pm 0.1$ & $9.5 \pm 0.2$ \\
\hline & Recovery (\%) & $25 \pm 15$ & $82 \pm 3$ & $78 \pm 3$ & $63 \pm 2$ & $36 \pm 1$ & $87 \pm 3$ & $93 \pm 6$ & $91 \pm 8$ & $90 \pm 6$ & $100 \pm 3$ & $94 \pm 3$ & $94 \pm 5$ & $93 \pm 3$ \\
\hline \multirow{4}{*}{$\begin{array}{l}\text { Sulfate } \\
\text { (mg/min) }\end{array}$} & Feed & $45.5 \pm 4$ & $27.4 \pm 2$ & $31.2 \pm 1.4$ & $30.3 \pm 1.3$ & $31.0 \pm 1.7$ & $25.3 \pm 2.7$ & $31.4 \pm 1.5$ & $34.2 \pm 4.4$ & $34.1 \pm 1.5$ & $31.2 \pm 2.0$ & $26.0 \pm 1.9$ & $33.6 \pm 1.8$ & $35.4 \pm 1.5$ \\
\hline & Desalinated & $1.2 \pm 0.2$ & $5.4 \pm 0.7$ & $2.2 \pm 0.4$ & $5.5 \pm 1.5$ & $5.3 \pm 1.5$ & $4.5 \pm 0.6$ & $0 \pm 0.1$ & $1.1 \pm 0.3$ & $0.5 \pm 0.1$ & $1.6 \pm 0.4$ & $1.0 \pm 0.4$ & $3.5 \pm 0.6$ & $4.2 \pm 0.5$ \\
\hline & Brine & $8.7 \pm 0.4$ & $10.0 \pm 1.1$ & $22.0 \pm 1.9$ & $13.6 \pm 0.8$ & $12.1 \pm 1.4$ & $17.6 \pm 1.8$ & $31.5 \pm 1.4$ & $29.8 \pm 1.2$ & $33.2 \pm 1.4$ & $24.3 \pm 1.7$ & $20.3 \pm 1.1$ & $26.2 \pm 1.5$ & $28.0 \pm 1.4$ \\
\hline & Recovery (\%) & $22 \pm 4$ & $56 \pm 9$ & $77 \pm 16$ & $63 \pm 18$ & $56 \pm 17$ & $88 \pm 17$ & $99 \pm 23$ & $90 \pm 22$ & $99 \pm 16$ & $83 \pm 20$ & $82 \pm 37$ & $89 \pm 16$ & $91 \pm 13$ \\
\hline \multirow{3}{*}{$\begin{array}{l}\mathrm{M} /(\mathrm{C}+\mathrm{S}) \\
\text { ratio }\end{array}$} & Feed & $2.20 \pm 0.09$ & $2.58 \pm 0.15$ & $2.43 \pm 0.19$ & $2.43 \pm 0.19$ & $2.43 \pm 0.19$ & $2.58 \pm 0.19$ & & & & $2.63 \pm 0.72$ & $2.61 \pm 0.15$ & $2.61 \pm 0.15$ & $2.61 \pm 0.15$ \\
\hline & Desalinated & $0.65 \pm 0.03$ & $0.44 \pm 0.09$ & $0.64 \pm 0.07$ & $0.36 \pm 0.08$ & $0.39 \pm 0.09$ & $0.37 \pm 0.06$ & & & & $0.72 \pm 0.08$ & $0.49 \pm 0.13$ & $0.24 \pm 0.09$ & $0.26 \pm 0.09$ \\
\hline & Brine & $2.45 \pm 0.07$ & $2.38 \pm 0.13$ & $2.76 \pm 0.18$ & $2.68 \pm 0.20$ & $2.75 \pm 0.22$ & $2.85 \pm 0.10$ & & & & $2.68 \pm 0.22$ & $2.75 \pm 0.17$ & $2.77 \pm 0.15$ & $2.83 \pm 0.16$ \\
\hline \multirow[t]{2}{*}{$\mathrm{pH}$} & Desalinated & - & 10.0 & - & - & - & 10.5 & 10.5 & 10.5 & 10.5 & 10.5 & 10.5 & 10.5 & 10.5 \\
\hline & Brine & - & 13.5 & - & - & - & 14 & 14 & 14 & 14 & 13.5 & 14 & 14 & 14 \\
\hline
\end{tabular}

3 
Table S2. Concentration of cations and anions (with the exception of hydroxide) in outlet effluents.

\begin{tabular}{|c|c|c|c|c|c|c|c|c|c|c|c|c|c|c|}
\hline \multirow[b]{2}{*}{ Element } & \multirow[b]{2}{*}{ Concentration } & \multicolumn{13}{|c|}{$\mathrm{K} /(\mathrm{K}+\mathrm{Na})$ Ratio } \\
\hline & & $\begin{array}{c}0 \\
(39 \min )\end{array}$ & $\begin{array}{c}0.1 \\
(86 \mathrm{~min})\end{array}$ & $\begin{array}{c}0.2 \\
(77 \mathrm{~min})\end{array}$ & $\begin{array}{c}0.2 \\
(154 \mathrm{~min})\end{array}$ & $\begin{array}{c}0.2 \\
(213 \mathrm{~min})\end{array}$ & $\begin{array}{c}0.25 \\
101(\mathrm{~min})\end{array}$ & $\begin{array}{c}0.4 \\
(75 \mathrm{~min})\end{array}$ & $\begin{array}{c}0.4 \\
(138 \mathrm{~min})\end{array}$ & $\begin{array}{c}0.4 \\
(300 \mathrm{~min})\end{array}$ & $\begin{array}{c}0.5 \\
(88 \mathrm{~min})\end{array}$ & $\begin{array}{c}0.75 \\
(90 \mathrm{~min})\end{array}$ & $\begin{array}{c}0.75 \\
(170 \mathrm{~min})\end{array}$ & $\begin{array}{c}0.75 \\
(225 \mathrm{~min})\end{array}$ \\
\hline \multirow{2}{*}{$\mathrm{Na}(\mathrm{ppm})$} & Desalinated & $232 \pm 25$ & $146 \pm 25$ & $99 \pm 1$ & $107 \pm 25$ & $113 \pm 25$ & $86 \pm 1$ & & & & $45 \pm 1$ & $9 \pm 1$ & $0 \pm 1$ & $16 \pm 1$ \\
\hline & Brine & $14557 \pm 25$ & $34981 \pm 50$ & $38384 \pm 50$ & $28899 \pm 25$ & $16700 \pm 25$ & $35460 \pm 50$ & & & & $22532 \pm 25$ & $10730 \pm 25$ & $11046 \pm 25$ & $12864 \pm 25$ \\
\hline \multirow{2}{*}{$\mathrm{K}(\mathrm{ppm})$} & Desalinated & - & $88 \pm 1$ & $120 \pm 25$ & $123 \pm 25$ & $125 \pm 25$ & $135 \pm 25$ & & & & $308 \pm 25$ & $255 \pm 25$ & $252 \pm 25$ & $274 \pm 25$ \\
\hline & Brine & - & $8100 \pm 25$ & $17611 \pm 25$ & $13484 \pm 25$ & $6981 \pm 25$ & $20631 \pm 25$ & & & & $36626 \pm 50$ & $46269 \pm 50$ & $47347 \pm 50$ & $55128 \pm 50$ \\
\hline \multirow[t]{2}{*}{$\mathrm{S}(\mathrm{wt} \%)$} & Desalinated & $\begin{array}{c}0.037 \pm \\
0.002\end{array}$ & $\begin{array}{c}0.061 \pm \\
0.003\end{array}$ & $\begin{array}{c}0.035 \pm \\
0.002\end{array}$ & $\begin{array}{c}0.093 \pm \\
0.007\end{array}$ & $\begin{array}{c}0.067 \pm \\
0.006\end{array}$ & $\begin{array}{c}0.061 \pm \\
0.003\end{array}$ & $\begin{array}{c}0.014 \pm \\
0.002\end{array}$ & $\begin{array}{c}0.022 \pm \\
0.005\end{array}$ & $\begin{array}{c}0.024 \pm \\
0.005\end{array}$ & $\begin{array}{c}0.029 \pm \\
0.002\end{array}$ & $\begin{array}{c}0.025 \pm \\
0.002\end{array}$ & $\begin{array}{c}0.063 \pm \\
0.007\end{array}$ & $\begin{array}{c}0.070 \pm \\
0.005\end{array}$ \\
\hline & Brine & $0.38 \pm 0.01$ & $1.13 \pm 0.06$ & $1.22 \pm 0.03$ & $0.94 \pm 0.01$ & $0.49 \pm 0.02$ & $1.23 \pm 0.06$ & $1.20 \pm 0.05$ & $1.20 \pm 0.02$ & $1.22 \pm 0.02$ & $1.06 \pm 0.03$ & $0.97 \pm 0.03$ & $1.00 \pm 0.03$ & 1. $16 \pm 0.03$ \\
\hline \multirow{2}{*}{$\mathrm{S}^{2-}(\mathrm{mg} / \mathrm{L})$} & Desalinated & $275 \pm 37$ & $230 \pm 10$ & $160 \pm 20$ & $200 \pm 20$ & $200 \pm 20$ & $220 \pm 10$ & $160 \pm 10$ & $140 \pm 10$ & $200 \pm 10$ & $140 \pm 10$ & $150 \pm 10$ & $170 \pm 10$ & $200 \pm 10$ \\
\hline & Brine & $2750 \pm 73$ & $8700 \pm 200$ & $8700 \pm 300$ & $7200 \pm 300$ & $3000 \pm 300$ & $8500 \pm 500$ & $6900 \pm 300$ & $7200 \pm 300$ & $6900 \pm 300$ & $7000 \pm 500$ & $6600 \pm 300$ & $6600 \pm 300$ & $7500 \pm 300$ \\
\hline \multirow[t]{2}{*}{$\mathrm{C}(\mathrm{wt} \%)$} & Desalinated & $\begin{array}{c}0.001 \pm \\
0.001\end{array}$ & $\begin{array}{c}0.0001 \pm \\
0.001\end{array}$ & $\begin{array}{c}0.001 \pm \\
0.001\end{array}$ & 0 & 0 & $\begin{array}{c}0.001 \pm \\
0.001\end{array}$ & $\begin{array}{c}0.011 \pm \\
0.005\end{array}$ & $\begin{array}{c}0.008 \pm \\
0.002\end{array}$ & $\begin{array}{c}0.008 \pm \\
0.002\end{array}$ & $\begin{array}{c}0.001 \pm \\
0.002\end{array}$ & $\begin{array}{c}0.008 \pm \\
0.001\end{array}$ & $\begin{array}{c}0.009 \pm \\
0.002\end{array}$ & $\begin{array}{c}0.010 \pm \\
0.002\end{array}$ \\
\hline & Brine & $0.14 \pm 0.01$ & $0.45 \pm 0.01$ & $0.45 \pm 0.02$ & $0.36 \pm 0.01$ & $0.21 \pm 0.01$ & $0.45 \pm 0.01$ & $0.54 \pm 0.01$ & $0.54 \pm 0.01$ & $0.53 \pm 0.02$ & $0.47 \pm 0.01$ & $0.36 \pm 0.01$ & $0.36 \pm 0.01$ & $0.42 \pm 0.01$ \\
\hline
\end{tabular}

